\title{
1 Stochastic activation and bistability in a Rab GTPase regulatory network.
}

2 Urban Bezeljak ${ }^{1}$, Hrushikesh Loya ${ }^{2}$, Beata Kaczmarek ${ }^{1}$, Timothy E. Saunders ${ }^{3 \#}$, Martin Loose ${ }^{1 \#}$.

$3 \quad{ }^{1}$ Institute for Science and Technology Austria (IST Austria), Klosterneuburg, Austria

$4 \quad 2$ Indian Institute of Technology, Bombay, India

$5{ }^{3}$ Mechanobiology Institute and Department of Biological Sciences, National University of Singapore,

6 Singapore

$8 \quad$ \#Authors for correspondence: M.L.: martin.loose@ist.ac.at; T.E.S., dbsste@nus.edu.sg

Abstract

11 Rab GTPases are the central regulators of intracellular traffic. Their function relies on a conformational change triggered by nucleotide exchange and hydrolysis. While this switch is well understood for an individual protein, how Rab GTPases collectively transition between states to generate a biochemical signal in space and time is unclear. Here, we combine in vitro reconstitution experiments with theoretical modeling to study a minimal Rab5 activation network. We find that positive feedback in this network gives rise to bistable switching of Rab5 activation and provide evidence that controlling the inactive population of Rab5 on the membrane can shape the network response. Together, our findings reveal new insights into the non-equilibrium properties and general principles of biochemical signaling networks underlying the spatiotemporal organization of the cell.

\section{Introduction}

21 Positive feedback is a core motif in biochemical circuits that can generate bistable behavior, where 22 the system can collectively switch between an ON and OFF state (1). Regulatory networks 23 incorporating positive feedback loops control various cellular processes, such as cell polarization (2), 24 oocyte maturation (3), and cell cycle progression (4). Positive feedback has also been proposed to be 25 important for the organization of membrane traffic by small GTPases (5-7). Despite such ubiquity, the 26 molecular events underlying the emergent properties of these networks are currently poorly 27 understood.

28 Small GTPases of the Rab family organize the eukaryotic endomembrane system by defining the 29 biochemical identities of organelles and directing membrane traffic between intracellular 30 compartments through vesicle formation, transport, docking, and fusion with the target organelle (8). 31 Arguably the best characterized Rab GTPase is Rab5, which controls the maturation of early endosomes towards the lysosomal system (9). Like all small GTPases, Rabs can exist in either an 
active GTP- or inactive GDP-bound state. Additionally, Rab GTPases possess one or two lipophilic geranylgeranyl chains on their C-terminal, which anchor them to the membrane surface (10). There, they recruit downstream effectors to orchestrate the vesicular flow. The transition between nucleotide states is controlled by guanine nucleotide exchange factors (GEFs) that catalyze exchange of GDP with GTP; and GTPase activating proteins (GAPs) catalyzing GTP hydrolysis (11). In their inactive GDP-bound state, the Rab GDP-dissociation inhibitor (GDI) extracts the Rab GTPase from the membrane and keeps it soluble in the cytoplasm (12). As a result, nucleotide exchange and hydrolysis drive dynamic cycling of the GTPase to and from the membrane. In the case of Rab5, the GEF Rabex5 forms a complex with the Rab5 effector Rabaptin5 (13). Consequently, Rab5 is thought to recruit its own activator to establish a positive feedback motif, which was proposed to result in its ultrasensitive activation (14) and membrane accumulation (13, 15-19). However, whether these molecular interactions can indeed lead to switch-like activation and collective membrane binding of Rab5 is not known (20).

The reason for this lack of understanding is that the characterization of small GTPase networks on a systems level has remained challenging. First, the inherent complexity of the living cell makes in vivo control over reaction conditions and precise experimental readouts challenging. Second, in contrast to the situation in vivo, activity studies performed in vitro commonly relied on proteins without their physiological geranylgeranyl modifications and were performed in the absence of the GDI and membranes $(17,21)$. Accordingly, these simplified experimental setups can lead to non-physiological activation dynamics (22). Lastly, the input-output relationship of the Rab GTPase activation switch in a biologically relevant setting is currently unknown as previous in vitro assays of Rab regulation did not address the non-equilibrium dynamics of small GTPases under cycling conditions $(15,23,24)$.

Here, we rebuild the dynamic network underlying Rab5 activation in vitro using a minimal set of purified components (Fig. 1 and S1): fluorescently labeled, prenylated Rab5 in complex with GDI; Rabex5:Rabaptin5; and biomimetic membranes. In combination with theoretical modeling, this experimental approach allowed us to assay Rab5 activation far from biochemical equilibrium and to study the mechanisms of collective Rab5 activation under controlled conditions.

\section{Results}

61 First, we set out to verify the activity of purified Rabex5:Rabaptin5 on Rab5[GDP] in complex with GDI.

62 We loaded lipid-modified Rab5 with the fluorescent GDP analog mant-GDP and used its fluorescence 63 intensity as a real-time readout of nucleotide exchange $(13,16,17)$. With $60 \mathrm{nM}$ GEF and in the 64 absence of membranes, we could not detect nucleotide exchange on $250 \mathrm{nM}$ Rab5[mant-GDP]:GDI. 65 However, we found robust activation in the presence of small unilamellar vesicles (SUVs) (Fig. S2), confirming that the phospholipid bilayer is essential for activation of the Rab:GDI complex $(25,26)$. 
67 To investigate the role of biological membranes for Rab5 activation, we utilized glass supported lipid bilayers (SLBs) as membrane substrates, combined with fluorescently labeled proteins and TIRF microscopy (Fig. 1A) (27). To recapitulate the intracellular pre-activation state, we first incubated the SLB with inactive CF488A-Rab5:GDI (500 nM), $0.5 \mathrm{mM}$ GTP and $0.05 \mathrm{mM}$ GDP. We included free GDI $(2 \mu \mathrm{M})$ to mimic cellular stoichiometric excess of RabGDI (28). We then initiated nucleotide exchange by adding $200 \mathrm{nM}$ Rabex5:Rabaptin5 and followed the fluorescence of CF488A-Rab5 on the membrane. Starting from low basal level of fluorescence on the membrane surface, the addition of the GEF complex produced a characteristic rise in fluorescence intensity until the signal saturated after about 40 minutes (Fig. 1B), consistent with an accumulation of Rab5[GTP] on the membrane. Accordingly, SLBs can act as a membrane substrate for prenylated Rab5, allowing us to follow its collective activation and membrane binding in real time.

Positive feedback regulation typically gives rise to sigmoidal signal-response curves (29). To test for the presence of positive feedback in the Rabex5:Rabaptin5:Rab5 activation network we recorded Rab5 membrane binding after adding increasing amounts of the GEF complex (Fig. 1C). Strikingly, we found that this titration resulted in an apparent two-state response profile: while there was no activation at GEF concentrations below $20 \mathrm{nM}$ even 150 minutes after Rabex5:Rabaptin5 injection, we found a 10- to 80-fold increase of fluorescence on the membrane with higher concentrations of Rabex5:Rabaptin5 (Fig. 1D). From the temporal activation curves, we extracted the relative maximal rate of Rab5 activation ( $\left.k_{\max }\right)$ as well as the time delay needed to reach this rate $\left(T_{i}\right)(30)$ (Fig. S3, Materials and Methods). High GEF complex concentrations (400 nM) gave rise to an immediate increase in Rab5 fluorescence intensity. At intermediate GEF concentrations, we observed nearly flat intensity profiles for up to 2 hours before collective Rab5 activation (Fig. 1E). At low GEF concentrations, we observed no response within the measurement window (orange circles, Fig. 1E). We also performed extended time recordings at $8 \mathrm{nM}$ GEF and saw no response even after up to 12 hours (Fig. S4). Interestingly, the temporal delays needed to reach half activation increased linearly with the inverse of GEF complex concentrations (Fig. 1E, inset). Despite different delay times, all activation profiles had a similar sigmoidal shape (Fig. S5). By plotting $k_{\text {max }}$ against GEF concentration, we found that nucleotide exchange showed high cooperativity (Fig. 1F) with a critical GEF concentration of around $28 \mathrm{nM}$, where we observed significant variations between the response curves, with some measurements having no significant response over the time course of the experiment. Below this point, no collective switching was detected, while higher GEF concentrations allowed for fast activation and Rab5 membrane accumulation, which gradually increased (17).

99 To better understand the dynamic response curves and the origin of the observed activation delays, 100 we constructed a model of the minimal reaction network, which includes cooperative activation due to 101 a direct interaction of Rab5[GTP] with its GEF complex (Fig. 1G, Supplementary Text). Precise details 
of this cooperative interaction are not known, so in the model we take a conservative approach whereby the positive feedback is relatively weak. Solving the model using the Gillespie algorithm to incorporate biochemical noise (stochasticity) in the reactions (31) produces similar dynamics and time delays to those observed experimentally (Fig. 1H-K). In the absence of stochasticity, the predicted response curves deviated from the experiments: (1) at early times the intensity profiles were not flat, unlike measured experimentally; and (2) near the critical Rab5 concentration ( $30 \mathrm{nM})$, the model cannot replicate the broad range of activation times (Fig. S6). We cannot discount potential variations (e.g. precise initial protein concentrations) between each experiment playing a role in the observed results. However, given the highly controlled nature of our reconstituted experiment, we expect these fluctuations to be small. Together, our experimental and theoretical results provide clear evidence for positive feedback within a minimal Rab activation network sufficient to generate switch-like,

113 ultrasensitive behavior. Furthermore, stochasticity is relevant for the system response near the critical 114 switching concentration.

115 What are the molecular interactions giving rise to the observed cooperativity? It has been proposed 116 that cooperative Rab5 activation is due to GTP-dependent, effector-mediated GEF recruitment (13, 117 15-19). Alternatively, direct binding of Rabex5 to the negatively charged membrane could also 118 enhance nucleotide exchange by retaining the GEF complex on the membrane (32). To test these 119 possibilities, we prepared $\Delta_{R B D}$ Rabaptin5, which lacks Rab5 binding domains (RBDs) (20); and $\triangle$ Rabex5, which misses putative membrane targeting motifs (16) (Fig. 2A). Of all GEF complex variants tested, we detected efficient Rab5 activation only for full length Rabex5:Rabaptin5 and

$122 \Delta$ Rabex5:Rabaptin5. In contrast, there was no collective activation in the absence of Rabaptin5

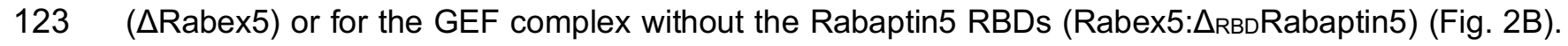
124 The same dependence on Rab5:Rabaptin5 interaction was also apparent in our model (Fig. 2C). Using 125 fluorescently labeled Rabaptin5 and dual color imaging, we found that Rab5 and the GEF complex showed similar intensity traces in experiments (Fig. 2D) and in our model (Fig. 2E), confirming that Rabex5:Rabaptin5 is retained on the membrane surface by active Rab5[GTP] to engage the positive feedback loop $(33,34)$. Together, these results demonstrate that Rabaptin5 not only enhances the GEF activity of Rabex5 $(17,35)$, but that direct interactions between GTPase, GEF and effector in a ternary complex are essential for the cooperative activation of Rab5 and its collective binding to the membrane (20).

What could explain the long delay times and stochastic switching observed at intermediate concentration of the GEF complex? Typically, long lag phases are related to processes that rely on random nucleation events that trigger phases of rapid growth $(36,37)$. Importantly, these lag phases

135 can be dramatically shortened in the presence of seeds that trigger activation. To test this prediction, 136 we attached different amounts of GTP-loaded constitutively active Rab5Q80L-His 10 on SLBs with 
nickel-chelating lipids (DOGS-NTA) before adding $80 \mathrm{nM}$ Rabex5:Rabaptin5 (Fig. 2F). Without preactivated Rab5 on the membrane (0 [DOGS-NTA]), activation occurred 20 min after addition of this concentration of Rabex5:Rabaptin5. In contrast, the time delays with Rab5Q80L-His 10 on $2 \%$ [DOGSNTA] membranes were 3-times shorter and completely absent with $5 \%$ [DOGS-NTA] (Fig. 2G), while the maximal activation rates were not significantly changed. This data shows that membrane-bound Rab5[GTP] can act as a seed for Rab5 activation and membrane accumulation.

Next, we wanted to find out what could initiate the Rab5 activation switch in the absence of active protein on the membrane. As the presence of membranes is required to activate the Rab5:GDI complex, we predicted that inactive Rab5[GDP] existing on the membrane prior to addition of the GEF complex is the substrate for nucleotide exchange $(22,38)$. Indeed, with small amounts of sCy5Rab5:GDI in a background of CF488A-labeled Rab5:GDI, we found individual sCy5-labeled proteins on the membrane even before adding Rabex5:Rabaptin5 (Fig. 3A). Using single molecule tracking, we found that non-activated sCy5-Rab5 diffused rapidly on the membrane and had a mean residence time of $0.3 \pm 0.1 \mathrm{~s}$ (Fig. 3B). After addition of the GEF complex, we found a sudden increase in sCy5Rab5 particle counts, along with a sigmoidal increase of membrane-bound CF488A-Rab5. The histogram of membrane residence times of Rab5[GTP] and corresponding fits revealed two populations: a short-lived population with a residence of $0.4 \pm 0.2 \mathrm{~s}$, similar to Rab5[GDP], and a longlived population with a 10-times longer residence time (3.3 $\pm 1.3 \mathrm{~s})$ (Fig. 3B). A similar membrane lifetime distribution was observed for Rab5 with the non-hydrolyzable GTP analog GMP-PNP (Fig. S7) indicating that the values for activated Rab5 are influenced by fluorophore bleaching and represent a lower bound of membrane-residence time. Together, these results indicate that Rab5 first transiently binds to the membrane in its GDP-bound state, before it is converted by Rabex5:Rabaptin5 to its longlived GTP-bound state. Rab5[GTP] on the membrane can then act as seed that retains GEF complex and initiates the positive feedback. Accordingly, initial random activation events are likely the cause of the observed stochasticity for its collective transition to the active state.

How do the initial levels of membrane-bound Rab5 and the strength of the positive feedback affect the transition between the ON and OFF states? To answer this question, we used a coarse-grained (phenomenological) version of our model, which incorporated only binding ( $\left.a_{0}\right)$ and unbinding $\left(a_{2}\right)$ of Rab5 $[R]$ on the membrane along with positive feedback ( $a_{1}$, with activation concentration $\left.K\right)$ (Fig. S8, Supplementary Text): $\frac{d[R]}{d t}=a_{0}+a_{1} \frac{[R]^{2}}{[R]^{2}+K^{2}}-a_{2}[R]$. The parameter space that leads to GTPase switching (Fig. 3C and S9) reveals that the switch response (i.e. the fold change in membrane-bound Rab5) after activation is small when the basal biding rate is set high. Conversely, if the basal binding rates are too low, the critical threshold for switching fails to occur, even with stochastic fluctuations. 
170 This reveals that the system switching is potentially highly tunable, and dependent on both the basal

171 binding rate and positive feedback strength.

172 To experimentally test the model predictions for how GTPase activation is tuned, we first varied the 173 rate of extraction of Rab5[GDP] by adding different amounts of free GDI in our experiments (Fig. 3D).

174 We found that increasing the stoichiometric GDI excess lowered the basal background fluorescence 175 prior to activation, prolonged activation delay times after GEF addition, and limited $k_{\max }$, consistent 176 with a decreased basal binding rate. Using our full model, we also see similar results when altering 177 the level of free GDI (Fig. 3E) confirming that high membrane extraction rates of Rab5[GDP] cause 178 long delay times and stochastic activation (39).

179 To increase basal Rab5 binding, we first replaced GTP in our experiment with GMP-PNP. As this GTP 180 analog inhibits Rab5's high intrinsic GTPase activity (40), it should prevent extraction of activated 181 Rab5 from the membrane and therefore lead to a more robust transition into the ON state. In 182 agreement with this prediction, we observed immediate collective Rab5 membrane binding after 183 adding $80 \mathrm{nM}$ GEF complex with GMP-PNP and $2 \mu \mathrm{M}$ GDI, while the delay time was more than 36 184 min when we used GTP (Fig. 3D, magenta curve). Preventing Rab5 membrane extraction in the full 185 model but keeping other parameters fixed, we see that our model displays similar behavior for the 186 GMP-PNP nucleotide exchange (Fig. 3E, magenta curve). Next, we added the Rab5-specific GDI 187 dissociation factor - PRA1 to our experiments, which has been suggested to accelerate the release of $188 \mathrm{Rab}[\mathrm{GDP}]$ from the GDI complex (41). Accordingly, it should also increase the basal GTPase binding 189 rate and facilitate the collective activation switch. Indeed, with PRA1 in the membrane, we observed 190 fast Rab5 activation with short delay times even at a Rabex5:Rabaptin5 concentration too low to 191 support Rab5 activation on PRA1-free membranes (8 nM) (Fig. 3F). These findings show that despite 192 not strictly required for Rab5 activation (38, 42), the presence of PRA1 in the endosomal membrane 193 can lower the threshold for positive feedback initiation, making collective Rab5 activation more likely.

194 Conversely, further increasing Rab5's GTPase activity above its intrinsic rate should inhibit collective 195 switching as it prevents effector recruitment of the GEF complex and facilitates extraction of Rab5 196 from the membrane (Fig. 3C; moving to the left along the red line). To test this prediction, we performed 197 experiments in the presence of purified full-length RabGAP-5 (SGSM3), a Rab5-specific GAP (43), 198 which stimulates GTP hydrolysis by Rab5. We recorded the signaling response after addition of 80 199 nM Rabex5:Rabaptin5 in the presence of increasing RabGAP-5 amounts (Fig. 4A) and found that 200 while it increased the activation delay, it did not substantially affect the maximal rate of Rab5 activation 201 (Fig. 4B and 4C). At RabGAP-5 concentrations between 100 and $250 \mathrm{nM}$ the reconstituted network 202 either showed successful activation events or no accumulation of Rab5 on the membrane for different 203 replicates at identical initial conditions. Importantly, once the system was switched ON, we found that 
even addition of $2 \mu \mathrm{M}$ GAP (Fig. 4D) does not completely reverse the system to its pre-activated state. Similarly, by increasing the dissociation rate $a_{2}$ in our phenomenological model, we observed clear difference in switching responses after 150 minutes, depending on the initial state of the network (Fig. $4 \mathrm{E})$. This hysteretic response of the system confirms the bistable behavior of the Rab5 activation network.

Strikingly, at RabGAP-5 concentrations of $50 \mathrm{nM}$, we observed Rab5 activation fronts on the membrane, where areas of high Rab5 density coexisted next to low Rab5 density areas (in 9 out of 13 experiments, in 3 experiments no obvious waves were noticed during activation, while in one experiment no activation occurred). This spatiotemporal activation pattern existed for more than 30 min, during which the activation front spread at a velocity of $5 \mu \mathrm{m} / \mathrm{min}$ before the system settled into a

214 fully active state with Rab5 covering the SLB at high density (Fig. 4F, G). What could explain the 215 emergence of this spatial pattern? Local activation of Rab5[GTP], due to random fluctuations, is 216 reinforced and stabilized by positive feedback via engagement of Rabex5:Rabaptin5. This region of 217 initial Rab5[GTP] activation will have higher probability of further Rab5[GTP] recruitment at its 218 boundary than elsewhere on the surface, giving rise to a propagating activation front. This emergent 219 property can be captured in our phenomenological model by introducing a diffusive term, where Rab5 220 activation in presence of a GAP can spread at constant rate by propagating the positive feedback 221 activation via an activation front (44). Such a front is dependent on the GAP activity and the threshold 222 Rab5[GTP] density that can sustain the positive feedback activation (Fig. 4H, Supplementary Text). It 223 is well known that dynamic biochemical systems composed of locally acting cooperative actuators and 224 long-ranged inactivators can give rise to chemical waves on the cellular and tissue level (45-48). In 225 our system, RabGAP-5 acts as a global inhibitor, rather than a long-ranged diffusing inhibitor, resulting 226 in our relatively simpler spatio-temporal patterns of activation.

\section{Discussion}

228 To summarize, using in vitro reconstitution and theoretical modeling, we found that the minimal Rab5 229 regulatory network is ultrasensitive and bistable, likely prerequisites for the decisive signaling reactions 230 controlling vesicle traffic. We have demonstrated that the architecture of the Rab5 activation network 231 supports the formation of spatiotemporal patterns such as activation fronts, as found for other bistable 232 systems with a local positive feedback and global inhibition (46). We also found that Rab5 activation 233 in this minimal network can occur stochastically, and we identified the low amounts of non-active 234 Rab5[GDP] as a potential source for this stochastic behavior. While stochasticity and long delay times 235 are generally disadvantageous for intracellular signaling reactions that rely on tight control, our in vitro experiments demonstrate that it is possible to tune the response of the Rab5 activation network by 
237 regulating the stability of the Rab:GDI complex, either by the presence of a GDF in the membrane and 238 possibly via GDI phosphoregulation (49).

239 Our study represents a systematic characterization of a minimal biochemical circuit of Rab GTPase 240 activation. We have also provided examples for how additional regulatory interactions can be 241 employed to direct and tune small GTPase activation in space and time. Of course, the composition 242 of the cell provides more complex modes of regulation, both at the protein and membrane levels. Our 243 in vitro system can be further extended to include other effectors or membrane compositions, making 244 it an excellent testbed for probing the mechanisms of organelle identity formation during vesicle 245 trafficking and the compartmentalization of the living cell. Furthermore, our approach can also be used 246 to study the dynamic networks of other small GTPase families, such as Arf, Rac and Rho GTPases.

\section{References}

249 1. T. Gardner, C. Cantor, J. Collins, Construction of a genetic toggle switch in Escherichia coli. $250 \quad$ Nature. 403, 339-342 (2000).

2512 2. S. J. Altschuler, S. B. Angenent, Y. Wang, L. F. Wu, On the spontaneous emergence of cell 252 polarity. Nature. 454, 886-9 (2008).

253 3. C. Y. Huang, J. E. Ferrell, Ultrasensitivity in the mitogen-activated protein kinase cascade. Proc. $254 \quad$ Natl. Acad. Sci. U. S. A. 93, 10078-83 (1996).

255 4. J. R. Pomerening, E. D. Sontag, J. E. Ferrell, Building a cell cycle oscillator: hysteresis and

5. M. Zerial, H. McBride, Rab proteins as membrane organizers. Nat. Rev. Mol. Cell Biol. 2, 107-

6. E. Mizuno-Yamasaki, F. Rivera-Molina, P. Novick, GTPase networks in membrane traffic.

7. F. A. Barr, Rab GTPases and membrane identity: Causal or inconsequential? J. Cell Biol. 202,

8. H. Stenmark, Rab GTPases as coordinators of vesicle traffic. Nat. Rev. Mol. Cell Biol. 10, 51325 (2009).

9. A. Zeigerer et al., Rab5 is necessary for the biogenesis of the endolysosomal system in vivo. Nature. 485, 465-70 (2012). 
10. A. Kalinin et al., Expression of mammalian geranylgeranyltransferase type-II in Escherichia coli and its application for in vitro prenylation of Rab proteins. Protein Expr. Purif. 22, 84-91 (2001).

11. J. Cherfils, M. Zeghouf, Regulation of small GTPases by GEFs, GAPs, and GDIs. Physiol. Rev. 93, 269-309 (2013).

271 12. K. Gavriljuk, A. Itzen, R. S. Goody, K. Gerwert, C. Kötting, Membrane extraction of Rab proteins by GDP dissociation inhibitor characterized using attenuated total reflection infrared spectroscopy. Proc. Natl. Acad. Sci. U. S. A. 110, 13380-5 (2013).

13. Z. Zhang et al., Molecular mechanism for Rabex-5 GEF activation by Rabaptin-5. Elife. 3, 119 (2014).

14. J. E. Ferrell, S. H. Ha, Ultrasensitivity part I: Michaelian responses and zero-order ultrasensitivity. Trends Biochem. Sci. 39, 496-503 (2014).

15. R. Lippé, M. Miaczynska, V. Rybin, A. Runge, M. Zerial, Functional synergy between Rab5 effector Rabaptin-5 and exchange factor Rabex-5 when physically associated in a complex. Mol. Biol. Cell. 12, 2219-28 (2001).

16. A. Delprato, E. Merithew, D. G. Lambright, Structure, exchange determinants, and family-wide Rab specificity of the tandem helical bundle and Vps9 domains of Rabex-5. Cell. 118, 607-617 (2004).

17. A. Delprato, D. G. Lambright, Structural basis for Rab GTPase activation by VPS9 domain exchange factors. Nat. Struct. Mol. Biol. 14, 406-12 (2007).

18. H. Stenmark, G. Vitale, O. Ullrich, M. Zerial, Rabaptin-5 is a direct effector of the small GTPase Rab5 in endocytic membrane fusion. Cell. 83, 423-32 (1995).

19. H. Horiuchi et al., A novel Rab5 GDP/GTP exchange factor complexed to Rabaptin-5 links nucleotide exchange to effector recruitment and function. Cell. 90, 1149-59 (1997).

20. S. Kälin, D. T. Hirschmann, D. P. Buser, M. Spiess, Rabaptin5 is recruited to endosomes by

292 21. L. Langemeyer et al., Diversity and plasticity in Rab GTPase nucleotide release mechanism has consequences for Rab activation and inactivation. Elife. 3, e01623 (2014). 
23. T. Ohya et al., Reconstitution of Rab- and SNARE-dependent membrane fusion by synthetic endosomes. Nature. 459, 1091-7 (2009).

24. L. Langemeyer, A. Perz, D. Kümmel, C. Ungermann, A guanine nucleotide exchange factor (GEF) limits Rab GTPase-driven membrane fusion. J. Biol. Chem. 293, 731-739 (2018).

25. L. L. Thomas, J. C. Fromme, GTPase cross talk regulates TRAPPII activation of Rab11 homologues during vesicle biogenesis. J. Cell Biol. 215, 499-513 (2016).

26. L. L. Thomas, S. A. van der Vegt, J. C. Fromme, A Steric Gating Mechanism Dictates the

27. P. a. Nguyen, C. M. Field, A. C. Groen, T. J. Mitchison, M. Loose, Using supported bilayers to study the spatiotemporal organization of membrane-bound proteins. Methods Cell Biol. 128,

28. S. R. Pfeffer, A. B. Dirac-Svejstrup, T. Soldati, Rab GDP dissociation inhibitor: putting rab

29. J. E. Ferrell, W. Xiong, Bistability in cell signaling: How to make continuous processes

30. K. M. C. Tjørve, E. Tjørve, The use of Gompertz models in growth analyses, and new

31. A. Landeros et al., BioSimulator.jl: Stochastic simulation in Julia. Comput. Methods Programs

32. H. Zhu et al., Rabaptin-5-independent membrane targeting and Rab5 activation by Rabex-5 in Gompertz-model approach: An addition to the Unified-Richards family. PLoS One. 12,

33. H. Zhu, H. Qian, G. Li, Delayed onset of positive feedback activation of Rab5 by Rabex-5 and Rabaptin-5 in endocytosis. PLoS One. 5, e9226 (2010).

34. A. Jilkine, S. B. Angenent, L. F. Wu, S. J. Altschuler, A density-dependent switch drives stochastic clustering and polarization of signaling molecules. PLoS Comput. Biol. 7, e1002271 (2011).

35. R. Lippe, H. Horiuchi, A. Runge, M. Zerial, Expression, purification, and characterization of Rab5 effector complex, Rabaptin-5/Rabex-5. Regul. Eff. Small Gtpases, Pt E. 329, 132-145 (2001). 
36. P. Arosio, T. P. J. Knowles, S. Linse, On the lag phase in amyloid fibril formation. Phys. Chem. Chem. Phys. 17, 7606-18 (2015).

37. E. Nishida, H. Sakai, Kinetic analysis of actin polymerization. J. Biochem. 93, 1011-20 (1983).

38. Y.-W. Wu et al., Membrane targeting mechanism of Rab GTPases elucidated by semisynthetic protein probes. Nat. Chem. Biol. 6, 534-40 (2010).

39. N. E. Buchler, F. R. Cross, Protein sequestration generates a flexible ultrasensitive response

40. V. Rybin et al., GTPase activity of Rab5 acts as a timer for endocytic membrane fusion. Nature. 383, 266-9 (1996).

41. U. Sivars, D. Aivazian, S. R. Pfeffer, Yip3 catalyses the dissociation of endosomal Rab-GDI complexes. Nature. 425, 856-9 (2003).

42. J. Blümer et al., RabGEFs are a major determinant for specific Rab membrane targeting. J. Cell Biol. 200, 287-300 (2013).

43. A. K. Haas, E. Fuchs, R. Kopajtich, F. A. Barr, A GTPase-activating protein controls Rab5

44. D. J. Jörg et al., The proneural wave in the Drosophila optic lobe is driven by an excitable reaction-diffusion mechanism. Elife. 8, 1-34 (2019).

344 45. Gierer, Meinhardt, A Theory of Biological Pattern Formation. Kybernetik. 12, 30-39 (1972).

46. V. E. Deneke, S. Di Talia, Chemical waves in cell and developmental biology. J. Cell Biol. 217, 1193-1204 (2018).

47. C. Brachmann, A. Davies, G. Cost, deletion strains derived from Saccharomyces cerevisiae S288C: a useful set of strains and plasmids for PCR-mediated gene disruption and other applications. YEAST- .... 132, 115-132 (1998).

48. A. M. Turing, The chemical basis of morphogenesis. Philos. Trans. R. Soc. Lond. B. Biol. Sci. of rab proteins. FEBS Lett. 329, 313-8 (1993). 
A

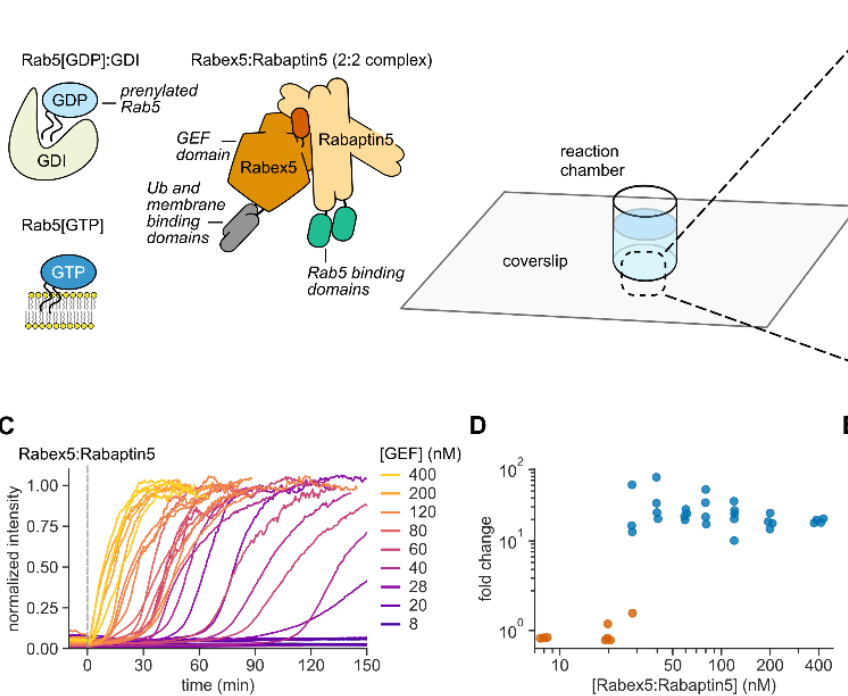

Rab5 activation in vitro reconstitution assay

$\mathbf{G}$

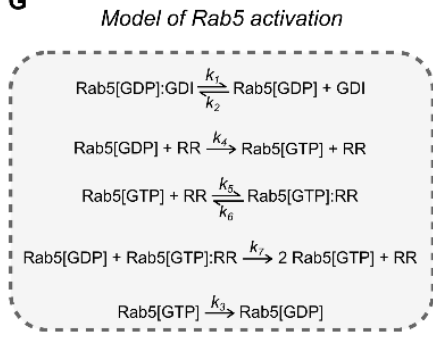

H

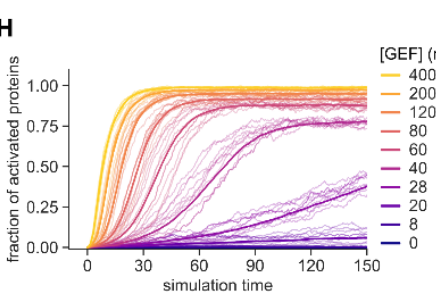

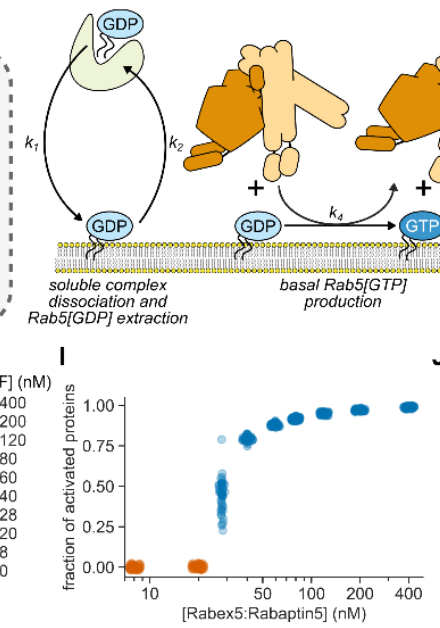
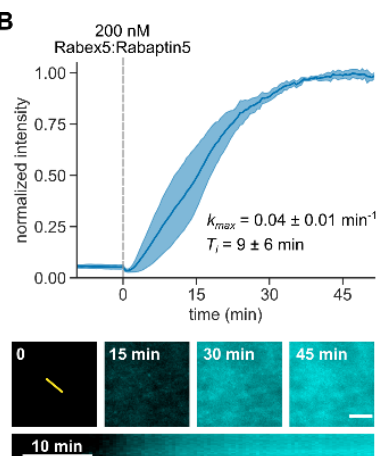

$\mathbf{F}$

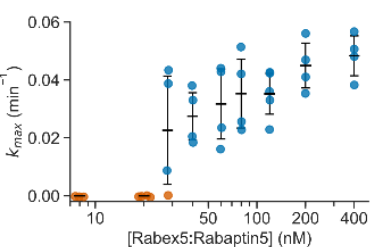

Fig. 1. Rab5:GDI activation on phospholipid membranes is ultrasensitive and stochastic.

(A) Schematic of Rab5 activation reconstitution assay on a supported lipid bilayer (SLB). (B) Top panel: addition of Rabex5:Rabaptin5 triggers nucleotide exchange by CF488A-Rab5, which can be followed by an increase of fluorescence intensity on the membrane surface. Solid line is mean normalized intensity, shaded area corresponds to SD $(n=4)$. Bottom: micrographs of CF488A-Rab5 binding to the SLB after addition of 200 nM GEF complex and corresponding kymograph (below) taken along the yellow line. Scale bar $=5 \mu \mathrm{m}$. (C) Rab5 intensity traces obtained at increasing Rabex5:Rabaptin5 concentrations. (D) Rab5:GDI- Rabex5:Rabaptin5 activation response curve. The fold change was calculated by dividing the fluorescence intensity at steady state with the average signal 10 min before GEF addition. (E) Activation delay $T_{i}$ decreases with higher Rabex5:Rabaptin5 concentration. Where no detectable activation was observed within $150 \mathrm{~min}$, the $T$ s are denoted as $>150 \mathrm{~min}$ and shown in orange. Error bars are SD. (F) Relative maximum rates $k_{\max }$ against the GEF complex concentration reveal cooperativity of Rab5 activation. Without detectable activation within 
$368150 \mathrm{~min}$, the activation rate was determined to be 0 and the corresponding points are depicted in 369 orange. Error bars are means \pm SD. (G) Schematic representation of modeled molecular interactions.

370 We constructed a model of the minimal Rab5 activation network based on the known literature

$371(13,15-19,38)$. We then derived ODEs based on mass action kinetics. (H) Stochastic model 372 simulations of Rab5 activation at increasing Rabex5:Rabaptin5 particle numbers. Shown are average

373 curves from 50 individual runs in bold and 10 random traces per condition. (I - K) Signal fold change,

374 temporal delays and relative maximum rates from the stochastic simulations in $(\mathrm{H})$. We ran 50

375 individual stochastic simulations per condition. 
A

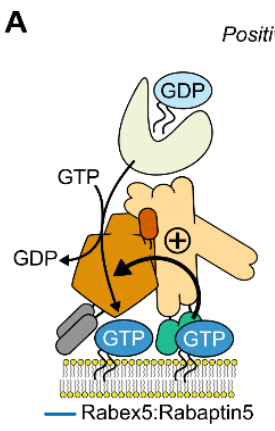

Positive feedback

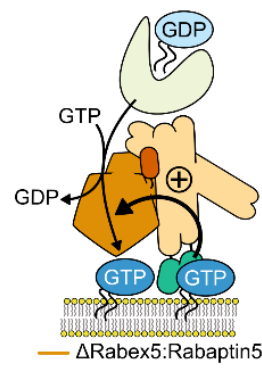

No positive feedback
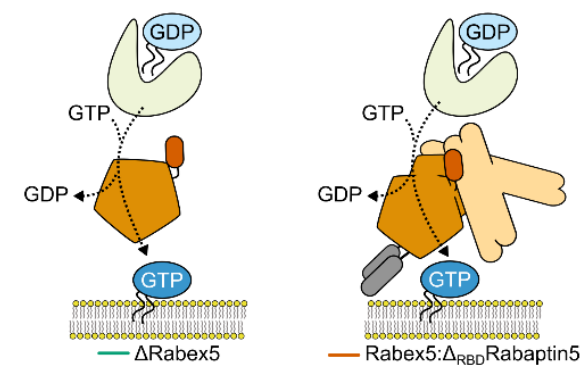

D Rabex5:Rabaptin5

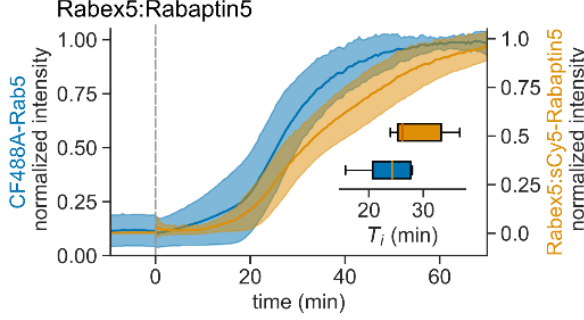

B

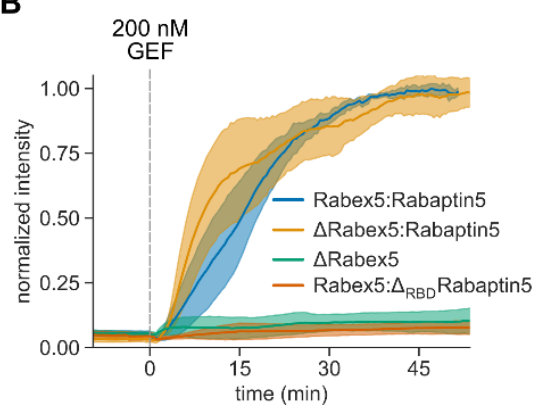

C

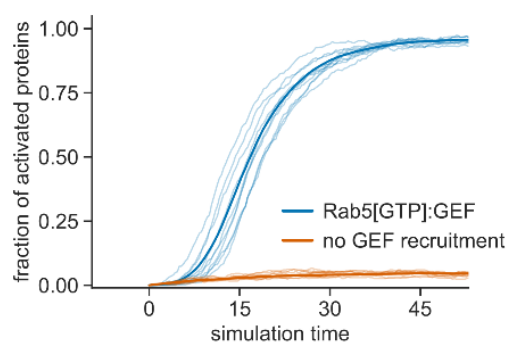

E

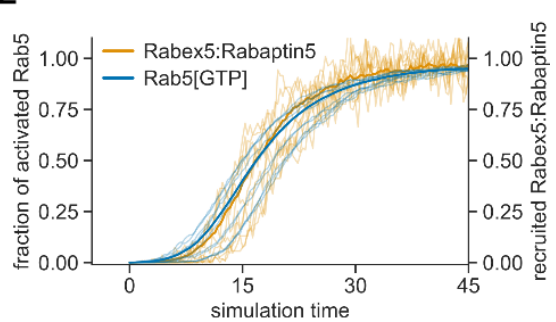

$\mathbf{F}$

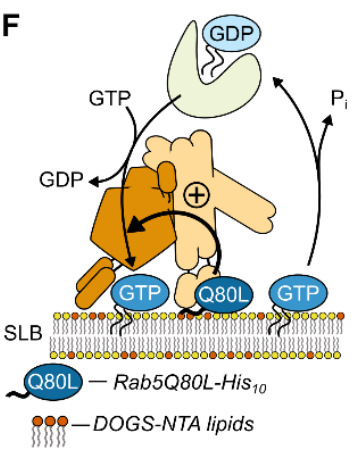

G

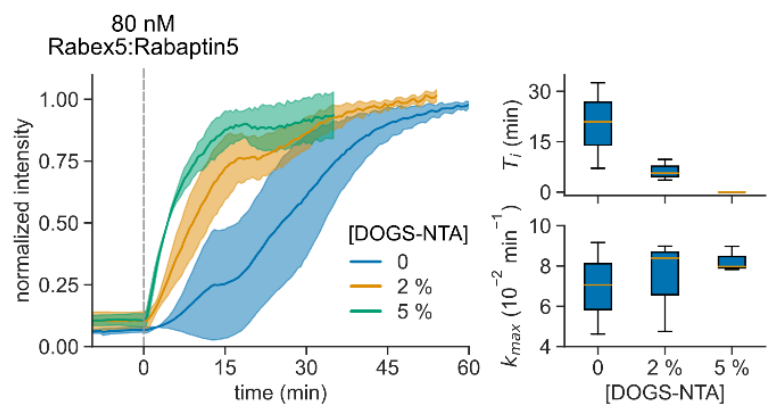

\section{Fig. 2. Positive feedback of Rab5 activation depends on GEF recruitment.}

(A) Illustration of protein interactions responsible for collective Rab5 switching. Positive feedback originates from a direct interaction between Rabex5:Rabaptin5 and Rab5[GTP]. (B) Fluorescence intensity traces obtained from experiments depicted in (A). Solid lines are mean normalized intensities, shaded areas are SD

(Rabex5:Rabaptin5,

$\Delta$ Rabex5:Rabaptin5

$\mathrm{n}=4 ; \Delta$ Rabex5, Rabex5: $\triangle$ RBDRabaptin5 $\mathrm{n}=3$ ).

(C) Stochastic model simulations with and without Rabex5:Rabaptin5:Rab5[GTP] complex formation $\left(k_{5}, k_{6}=0\right)$ for 200 Rabex5:Rabaptin5 particles. Average curves from 50 individual runs are depicted in bold with 10 random traces per condition. (D) 
386 normalized fluorescence intensity, shaded area is SD $(n=5)$. Inset: $T_{i}$ for CF488A-Rab5 (blue) and 387 Rabex5:sCy5-Rabaptin5 (orange). (E) Stochastic model simulations for Rab5 and Rabex5:Rabaptin5 388 membrane binding for 200 Rabex5:Rabaptin5 particles. Shown are curves from 50 independent runs, 389 the mean line is depicted bold with 10 random traces per condition. (F) Schematic of the reconstitution 390 experiment with pre-activated SLB-immobilized Rab5Q80L-His ${ }_{10}[G T P]$. (G) Collective switching is 391 faster with pre-activated Rab5. Left: Rab5 switching time courses in presence of 500 nM Rab5Q80L392 His 10 with increasing DOGS-NTA lipid concentration in the SLB. Solid line is mean normalized 393 fluorescence intensity over time, shaded area is mean \pm SD $(n=3)$. Right: corresponding time delays $394 \quad T_{i}$ and relative maximum rates $k_{\max }$ 
A

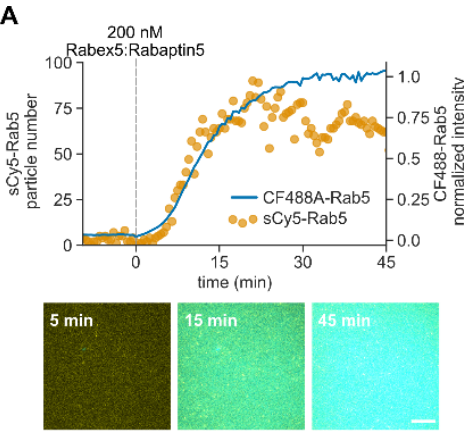

D

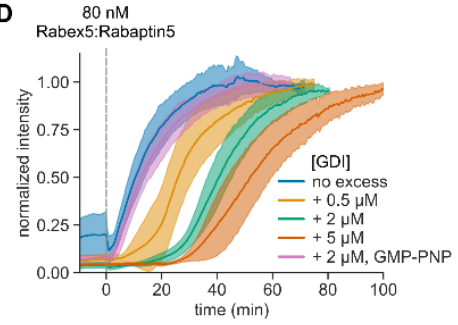

B

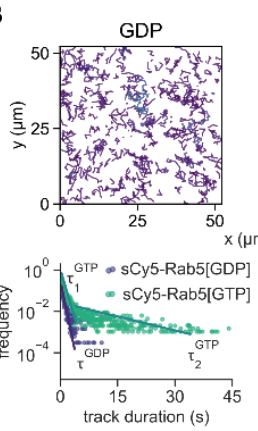

$E$
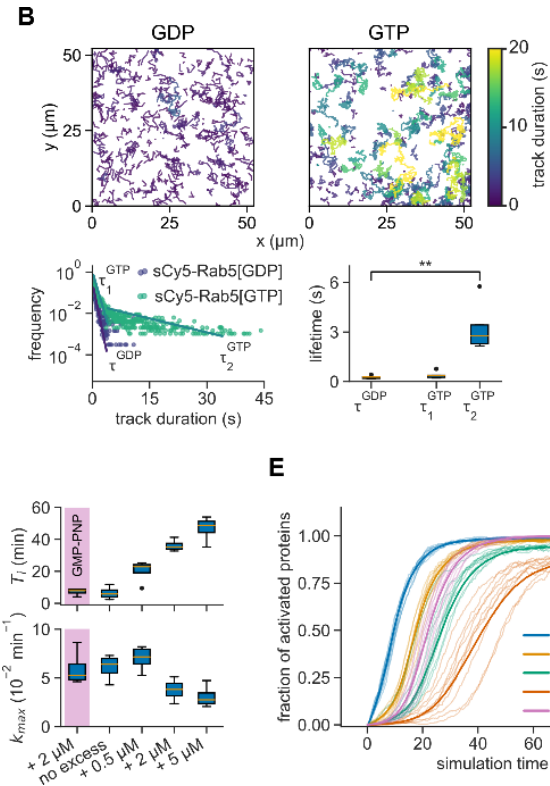

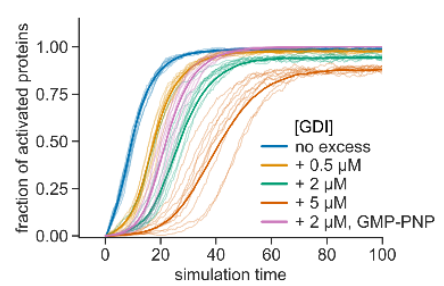

C

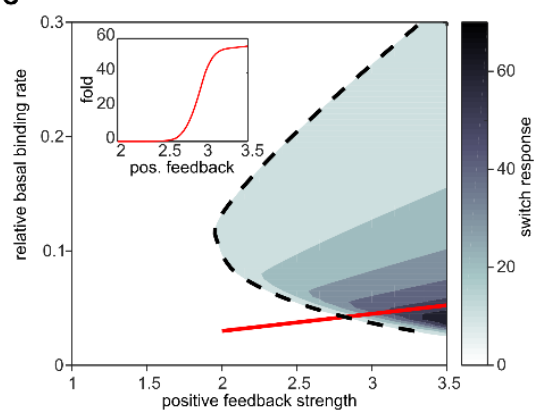

F $8 \mathrm{nM}$

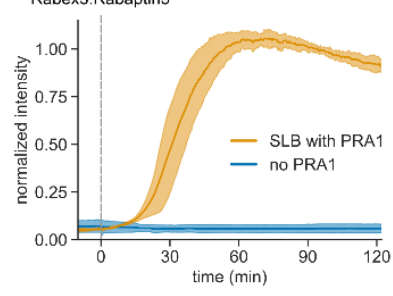

Fig. 3. Rab5:GDI activation is tuned by free Rab5[GDP] abundance.

(A) Rab5 cycles between the membrane and solution before and after nucleotide exchange. Top: sCy5-Rab5 molecule counts per frame and collective CF488A-Rab5 activation. Bottom: snapshots of the activation reaction. SCy5- and CF488A-Rab5 are depicted in yellow and cyan, respectively. Scale bar is $10 \mu \mathrm{m}$. (B) Rab5 single molecule trajectories reveal GDP- and GTP-bound proteins on the membrane. Top: 500 tracks of membrane-bound sCy5-Rab5 particles before (GDP) and after (GTP) activation. Bottom: frequency histogram identifies two populations with distinct lifetimes. A monoexponential decay before activation with lifetime $T^{\mathrm{GDP}}$ and two-exponential decay with lifetimes $\mathrm{T}_{1}{ }^{\mathrm{GTP}}$ and $\mathrm{T}_{2}{ }^{\mathrm{GTP}}$, respectively $(\mathrm{n}=5)$. (C)

(C) Parameter phase space of the phenomenological model for Rab5 switching, depending on the basal rate of activation $\left(a_{0} / a_{2} K\right)$ and the strength of positive feedback $\left(a_{1} / a_{2} K\right)$. Switching is defined as the relative difference in steady-state concentration relative to the scenario with no positive feedback. Inset: fold activation along the red line in the diagram. Stochasticity introduced by solving the phenomenological model within a Fokker-Planck framework. See text for parameter definitions. (D) Stoichiometric GDI excess over Rab5 affects delay of Rab5 activation in vitro. Left: solid lines are mean normalized intensities over time, shaded areas correspond to SD $(n=3)$. Right: corresponding activation $T_{i}$ and relative maximum rates $k_{\max }$.

(E) Stochastic

412 simulations of the full model for varying initial amounts of GDI excess ( $0-2000$ particle number).

413 Shown are curves from 10 random runs per condition, the mean line from 50 runs is depicted bold. (F)

414 SLB-bound PRA1 enhances Rab5 activation at low GEF concentrations. Solid lines are mean 415 normalized fluorescence intensities, shaded areas correspond to SD $(n=3)$. 
A
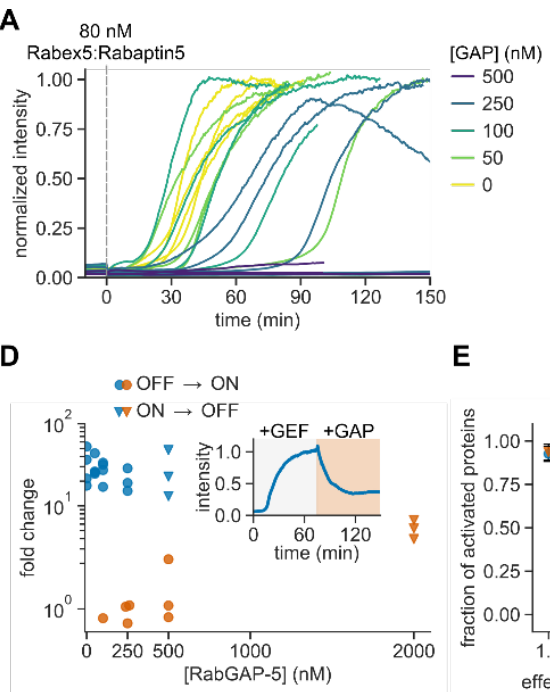

G

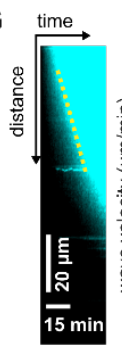

H
E
B

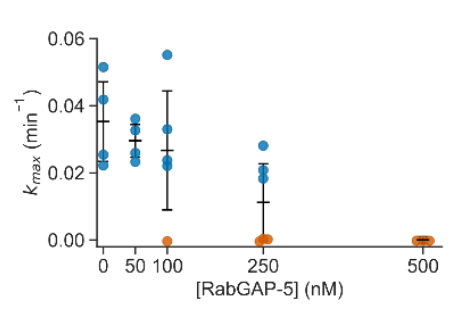

C

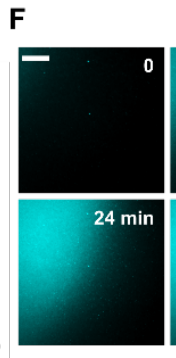

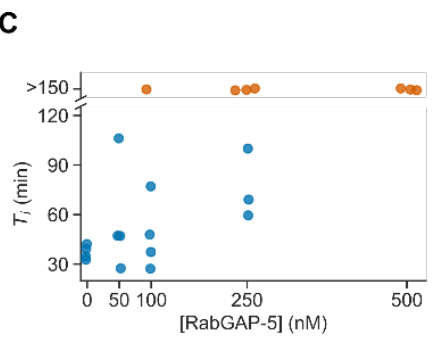

- OFF $\rightarrow$ ON
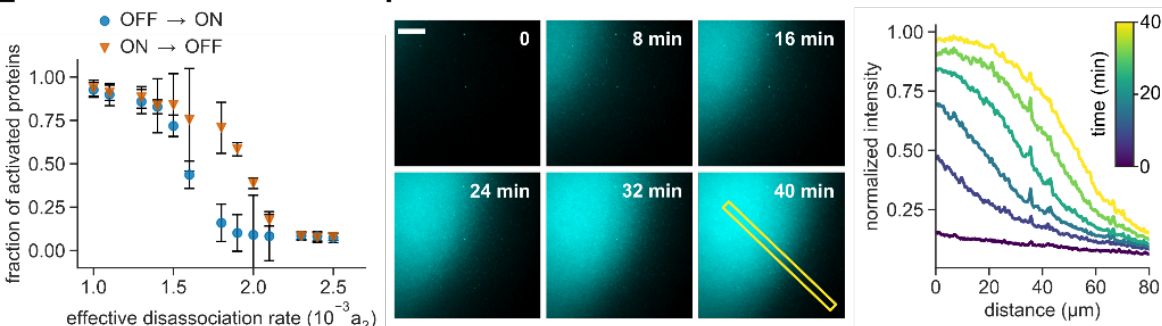

I

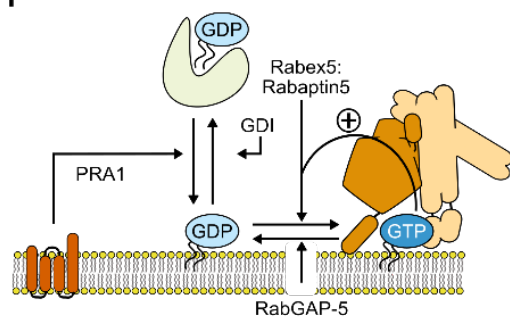

Fig. 4. GAP activity reveals bistability of the reconstituted network.

418 (A) Effect of RabGAP-5 on Rab5 activation. Shown are time courses at increasing GAP concentrations. (B) Maximal rates $k_{\max }$ of Rab5 activation for curves shown in (A). Without detectable activation within $150 \mathrm{~min}$, the activation rate was set to 0 and the corresponding points are depicted in orange. Error bars are SD.

(C) Activation delay $T_{i}$ for data presented in (A). Without detectable activation, the times to inflection point are denoted as $>150$ min (orange).

(D) GAP titration response curve. The fold change was calculated by dividing the fluorescence intensity at steady state with the average fluorescence signal 10 min before GEF addition. For ON $\rightarrow$ OFF switching, the system first reached active state (ON) with $80 \mathrm{nM}$ GEF. Then, RabGAP- 5 was added and the reaction was followed until the system reached a new steady state (OFF). Inset: ON $\rightarrow$ OFF switching time course with $2 \mu \mathrm{M}$ RabGAP-5. (E) Changing the dissociation rate reveals hysteresis in switching of the phenomenological model after 150 minutes. Shown are means of 20 simulations, error bars are \pm SD. (F) Left: Rab5 activation wave spreading across the SLB. Scale bar is $20 \mu \mathrm{m}$. Times indicate relative duration after start of acquisition, not time after addition of GEF complex. Right: fluorescence intensity profile of the indicated area. (G) Kymograph of the indicated area in $(F)$ and mean wave velocity. Wave velocity was determined from the slope of fluorescence increase in generated kymographs $(n=6)$. $(H)$

433 Simulated Rab5 activation front from including diffusion, $D$, within the phenomenological model; see 434 Supplementary Text Eq. 6. Solution in terms of the dimensionless distance $\frac{x}{\sqrt{D / a_{2}}}$. (I) Overview of the 435 reconstituted Rab5 network regulation. 


\section{Acknowledgments}

437 The authors sincerely thank B. Simons, K. Kruse, M. Howard, E. Hannezo, A. Yap, T. Lecuit, and J.

438 Brugués for discussions and valuable feedback on the manuscript. Additionally, we thank K. Loibl, 439 other Loose lab members and the Scientific Service Units at the IST Austria for their support. Funding:

440 this work was supported by the Human Frontier Science Program (HFSP YIP 4193) as well as the 441 European Research Council (ERC StG 679239). T.E.S. is grateful to the Kavli Institute, Santa Barbara,

442 which supported his visit during part of the manuscript preparation (supported in part by NSF Grant 443 No. PHY-1748958, NIH Grant No. R25GM067110, and the Gordon and Betty Moore Foundation Grant 444 No. 2919.01). Author contributions: Conceptualization, M.L.; Methodology, M.L., T.E.S. and U.B.; 445 Software, T.E.S. and H.L.; Validation, U.B., H.L., T.E.S.; Formal Analysis, U.B., H.L. and T.E.S.; 446 Investigation, U.B., B.K., H.L. and T.E.S.; Resources, U.B., B.K., H.L. and T.E.S.; Data Curation, U.B., 447 H.L., T.E.S.; Writing - Original Draft, M.L., T.E.S. and U.B.; Writing - Review \& Editing, U.B., H.L., B.K., 448 T.E.S., M.L.; Visualization, U.B. and T.E.S.; Supervision, M.L. and T.E.S.; Project Administration, M.L. 449 and T.E.S.; Funding Acquisition, T.E.S. and M.L. Competing interests: the authors declare no 450 competing interests. Data and materials availability: all data needed to evaluate and reproduce the 451 reported conclusions is available in the manuscript or the supplementary materials.

\section{List of Supplementary Materials}

454 Materials and Methods

455 Supplementary Text

456 Figs. S1 to S9

457 Tables S1 to S3

458 References $(50-61)$

459 Movies S1 to S6 